\title{
On gas flow effects in 3D edge transport simulations for DIII-D plasmas with resonant magnetic perturbations
}

\author{
H Frerichs ${ }^{1}$, D Reiter ${ }^{1}$, O Schmitz ${ }^{1}$, D Harting ${ }^{1}$, T E Evans ${ }^{2}$, Y \\ Feng $^{3}$ \\ ${ }^{1}$ Institute of Energy and Climate Research - Plasma Physics, Forschungszentrum \\ Jülich GmbH, Association EURATOM-FZJ, Partner in the Trilateral Euregio \\ Cluster, Jülich, Germany \\ ${ }^{2}$ General Atomics, San Diego, California, CA, USA \\ ${ }^{3}$ Max-Planck Institute for Plasma Physics, Greifswald, Germany \\ E-mail: h.frerichs@fz-juelich.de
}

\begin{abstract}
Pumping of neutral gas and re-fuelling in other locations (e.g. neutral beam injection, gas puffing) is a key ingredient in divertor physics. However, this has not been included in previous 3D edge plasma transport simulations with the EMC3-EIRENE code. Including these effects brings the simulations closer to reality and we demonstrate that this has a significant impact on divertor parameters. In particular, we study the impact of the pumping efficiency on ITER similar shape plasmas at the DIII-D tokamak in the presence of resonant magnetic perturbations (RMPs). Further, we investigate the so called "particle pump-out effect". We show that in the present transport model and for given pumping and re-fuelling rates, the density at the transition to the core region decreases with increasing perturbation current, but the temperature as well. The striation patterns in the target particle and heat fluxes are extended into the regular SOL, which is caused by cross-field transport into a thin layer around the perturbed separatrix.
\end{abstract}

PACS numbers: 52.65.-y, 52.65.Kj

(Some figures in this article are in colour only in the electronic version)

Submitted to: Nucl. Fusion

\section{Introduction}

Computer simulations are an important tool to quantify the controlling processes in ITER relevant edge plasmas. In particular, three dimensional simulations are required to investigate the effects of non-axisymmetric edge plasma configurations, which are of interest for certain scenarios at ITER and current fusion devices. The (in 2D edge transport codes often assumed) axisymmetry is explicitly broken in these scenarios by the application of external resonant magnetic perturbations (RMPs). While typically 
high-confinement (H-mode) plasmas are accompanied by periodic bursts of particle and energy fluxes at the plasma edge (the so called edge localized mode or ELM [1]), it has been found at the DIII-D [2, 3] and JET [4] tokamaks that the application of RMPs is a promising technique to control ELMs. ELM control is an important issue for ITER, because extrapolations from current experiments indicate that the otherwise resulting high transient heat loads to the first wall and the divertor targets would lead to a significant reduction of the lifetime of some important ITER wall components [5]. Hence, a 3D transport model for the plasma edge is required in order to advance the understanding of ITER relevant plasma physics including RMP application.

The EMC3-EIRENE code is such a tool $[6,7,8,9]$. It provides a self-consistent fluid treatment of particle, parallel momentum and electron and ion energy transport for the plasma as well as recycling and kinetic neutral particle transport. It is regularly applied to stellarator configurations such as W7-AS, W7-X and LHD $[10,11,12,13]$ and, since resent years, also to tokamak configurations. These latter include limiter configurations e.g. at TEXTOR $[14,15,16]$ or DIII-D [17], the ITER start-up configuration [18], and very recently also poloidal divertor configurations at DIII-D [19, 20, 21], JET [22] and AUG [23, 24].

Pumping of neutral gas and re-fuelling in other locations (e.g. neutral beam injection, gas puffing) is a key ingredient in divertor physics. While this is a common feature in 2D edge plasma simulations, no such effects have been explicitly included in all previous 3D edge plasma transport simulations with the EMC3-EIRENE code. However, we found that these effects are essential for simulations with high, H-mode like densities: the complete absense of additional re-fuelling sources results in flat density profiles in the confined region [21] (both for the RMP and unperturbed case) in contradiction to experimental observations.

We briefly summarize the EMC3 edge plasma transport model in section 2 and continue with a more detailed description of the upgraded particle balance scheme in the code including pumping and re-fuelling. The explicit accounting for neutral gas pumping results in a new free model parameter, the pumping efficiency $\varepsilon_{\text {pump }}$. We will study the impact of this parameter on an ITER similar shape (ISS) plasma at DIII-D in the presence of RMPs in section 3. Using the re-fuelling and pumping rates as boundary conditions allows to study the impact of an increasing perturbation current under fixed external conditions. This is investigated in section 4.

\section{A 3D model for the plasma edge}

The EMC3-EIRENE code is a coupled version of the edge plasma transport code EMC3 and the neutral particle transport code EIRENE. The EMC3 code solves a set of steady state fluid equations in a 3D space of essentially arbitrary magnetic field geometry:

\section{Plasma density $n$}

$\nabla \cdot\left[n u_{\|} \mathbf{e}_{\|}-D_{\perp} \underline{\mathbf{I}}_{\perp} \cdot \nabla n\right]=S_{p}$ 
On gas flow effects in $3 D$ edge transport simulations for DIII-D plasmas with resonant magnetic perturbation

\section{Parallel flow $u_{\|}$}

$$
\begin{gathered}
\nabla \cdot \mathbf{e}_{\|}\left[m_{i} n u_{\|}^{2}-\eta \mathbf{e}_{\|} \cdot \nabla u_{\|}\right]-\nabla \cdot \underline{\mathbf{I}}_{\perp} \cdot D_{\perp} \nabla\left(m_{i} n u_{\|}\right) \\
=-\mathbf{e}_{\|} \cdot \nabla p+S_{m}
\end{gathered}
$$

Electron temperature $T_{e}$

$$
\begin{gathered}
\nabla \cdot \mathbf{e}_{\|}\left[\frac{5}{2} T_{e} n u_{\|}-\kappa_{e} \mathbf{e}_{\|} \cdot \nabla T_{e}\right]-\nabla \cdot \underline{\mathbf{I}}_{\perp} \cdot\left[\chi_{e} n \nabla T_{e}+\frac{5}{2} T_{e} D_{\perp} \nabla n\right] \\
=-k\left(T_{e}-T_{i}\right)+S_{e e}
\end{gathered}
$$

Ion temperature $T_{i}$

$$
\begin{gathered}
\nabla \cdot \mathbf{e}_{\|}\left[\frac{5}{2} T_{i} n u_{\|}-\kappa_{i} \mathbf{e}_{\|} \cdot \nabla T_{i}\right]-\nabla \cdot \underline{\mathbf{I}}_{\perp} \cdot\left[\chi_{i} n \nabla T_{i}+\frac{5}{2} T_{i} D_{\perp} \nabla n\right] \\
=+k\left(T_{e}-T_{i}\right)+S_{e i}
\end{gathered}
$$

where $\mathbf{e}_{\|}$is the unit vector in direction of the magnetic field, $\underline{\mathbf{I}}_{\perp}=\underline{\mathbf{I}}-\mathbf{e}_{\|} \mathbf{e}_{\|}$the "cross-field tensor" with the unit tensor $\underline{\mathbf{I}}$ and $p=n\left(T_{e}+T_{i}\right)$ the static pressure. Parallel transport coefficients $\eta, \kappa_{e}, \kappa_{i}$ are taken from the classical transport theory by Braginskii [25], while anomalous cross-field transport is taken into account by free model parameters $D_{\perp}, \chi_{e}, \chi_{i}$, and fixed viscosity $\eta_{\perp}=m_{i} n D_{\perp}$. Interactions with neutral particles are taken into account by the sources $S_{p}, S_{m}, S_{e e}$ and $S_{e i}$, which are calculated by the kinetic transport code EIRENE. An iterative procedure is applied to obtain a self-consistent solution for all plasma and neutral particle parameters.

\subsection{Details of the particle balance}

In order to solve the set of partial differential equations (1)-(4), boundary conditions need to be specified. In the following we focus on the boundary conditions for the steady state particle balance in the EMC3 code. A sketch of the simulation domain is given in figure 1, demonstrating the application of different types of boundary conditions at different parts of the simulation boundary:

- Walls, in particular the divertor target, are a plasma sink. The plasma flux $\Gamma_{\text {target }}$ to the divertor target is "recycled" as neutral gas flux $\Gamma_{\text {rec }}=\Gamma_{\text {target }}$, which is given to the EIRENE code. The EIRENE code then calculates the transport of neutral particles until ionization occurs, either in the plasma simulation domain resulting in volumetric plasma sources $S_{p}$, or in the core region $\left(\Gamma_{\text {core }}\right)$ :

$$
\Gamma_{\text {rec }}=\int_{V_{\text {sim }}} d V S_{p}+\Gamma_{\text {core }} .
$$

- At the inner simulation boundary (ISB), plasma surface sources are given by the steady state ionization rate of neutral particles in the core: $\Gamma_{\text {ISB }}=\Gamma_{\text {core }}$. The density $n_{\mathrm{ISB}}$ can either be given directly as code input (which is implemented by an internal rescaling of $\Gamma_{\text {rec }}$ ), or the recycling flux $\Gamma_{\text {rec }}$ can be set manually. 


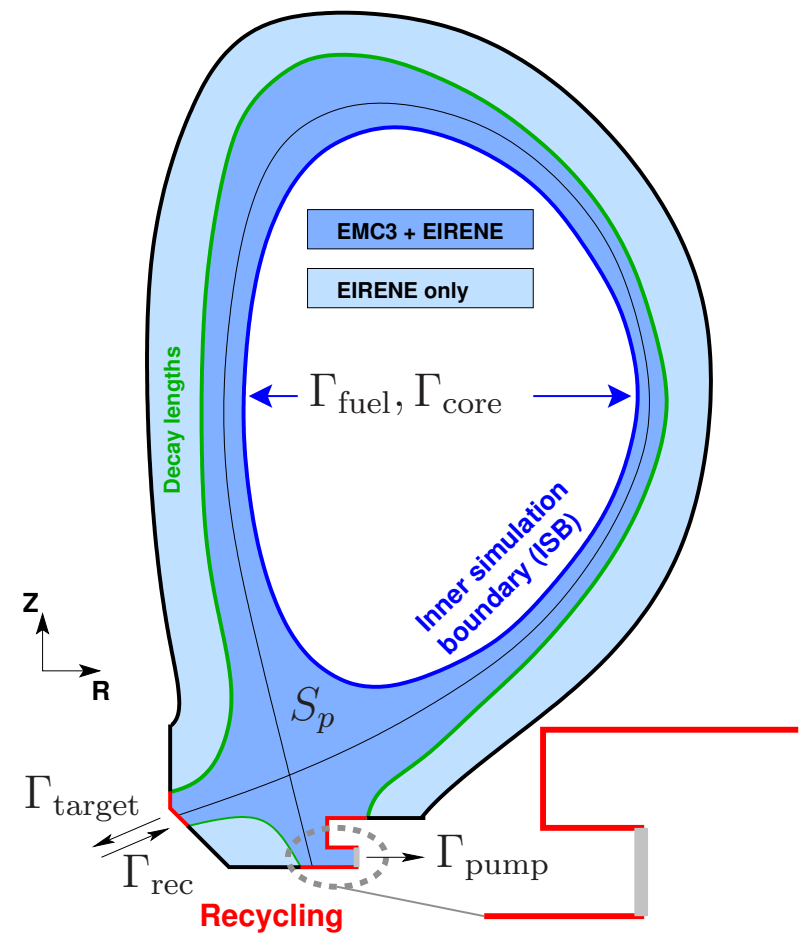

Figure 1. Sketch of a simulation domain for the EMC3-EIRENE code (poloidal cut at some arbitrary toroidal position). The position of different types of boundary conditions are colour-coded: recycling (red), in-fluxes (blue), e-folding length (green). The simulation domain for neutral particles (EIRENE) is extended up to the first wall.

- A radial e-folding length $\lambda_{p}$ is assumed at the outer simulation boundary. However, if this boundary is far enough outside, then the particle flux through this surface is negligible.

Boundary conditions are applied for the energy balance as well: sheath heat transmission coefficients at the targets, total power input through the inner simulation boundary and an e-folding length for the heat flux through the outer simulation boundary.

The particle balance in the experiment is controlled by neutral gas pumping and re-fuelling (e.g. gas puffing or neutral beam injection). Hence, in order to be closer to experimental conditions, we also introduce neutral gas pumping and re-fuelling in the code. At this point, we consider only "deep" re-fuelling, i.e. the re-fuelled neutral particles reach the core region through the inner simulation boundary (which is at $\Psi_{N} \approx 0.78$ in the present case), are ionized and re-enter the edge region as plasma sources $\Gamma_{\text {fuel }}$ on this surface. This is reasonable approximation only for fuelling by neutral beam injection (NBI). A more realistic treatment by additional volumetic sources, which is required in particular for re-fuelling by gas puffing, is not yet implemented in the code. This will probably modify the simulations results and will be investigated in the future.

In steady state, and under the assumption of perfect recycling (i.e. no wall retention), re-fuelling rate and pumped neutral gas flux are equal $\Gamma_{\text {pump }}=\Gamma_{\text {fuel }}$. This 
flux can be given as an alternative boundary condition in the new operation mode of the code. Hence, it allows to study properties of the perturbed plasma edge, in particular the particle pump-out effect, while external sources $\left(\Gamma_{\text {fuel }}, P_{\text {heat }}\right)$ are kept constant.

Pumping of neutral particles is implemented by a partially absorbing surface in the pump duct (grey surface in figure 1). This implementation results in a new model parameter: the pumping efficiency $\varepsilon_{\text {pump }}$, i.e. the absorption probability for particles which hit the selected surface. We will study the impact of this parameter in section 3 . Note that $\varepsilon_{\text {pump }}$ is not equal to the ratio of pumped flux to recycling flux $\Gamma_{\text {pump }} / \Gamma_{\text {rec }}$, which is sometimes referred to as pumping efficiency as well. While the latter depends on the position of the strike point, the former can be regarded as a quality parameter of the pump itself (i.e. it only determines what happens to neutral particles which are already in the pump duct).

\section{Impact of the pumping efficiency parameter}

Before we investigate the impact of the pumping efficiency parameter $\varepsilon_{\text {pump }}$ on an ITER similar shape plasma at DIII-D, we start with short description of the magnetic field structure which is used as an input for the plasma transport simulations.

\subsection{The magnetic field structure}

The perturbed magnetic field is considered within the so-called "vacuum approach", i.e. the modification of the magnetic field by a plasma response is neglected at this point. As already mentioned in [20], analysis of the validity of this approach is still ongoing.

We focus on DIII-D discharge 132731 at $t=2750 \mathrm{~ms}$ with a perturbation current of $I_{c}=4 \mathrm{kA}$ applied in the I-coils (a set of 6 upper and 6 lower coils located on the low field side) in a configuration with toroidal mode number $n=3$ in even parity. This H-mode discharge is characterized by a plasma current of about $I_{p}=1.5 \mathrm{MA}$ and a toroidal field of $B_{t}=1.8 \mathrm{~T}$ at the magnetic axis, resulting in an edge safety factor of $q_{95}=3.6$. Time traces of characteristic parameters of this discharge can be found in figure 1 (a) in [31].

The resulting magnetic field structure is visualized in figure 2. The complex field structure at the plasma edge includes magnetic island chains and domains with chaotic field line behavior. A helical lobe structure is formed by the perturbed separatrix (i.e. the separatrix manifolds, also known as homoclinic tangle [26, 27]) which results in a striation pattern at the inner and outer strike points. These helical lobes guide field lines from the plasma interior to the wall, resulting in enhanced outward plasma transport along open field lines. Furthermore, the striation pattern is extended into the regular scrape-off layer (SOL, i.e. the short field line connection length region outside the separatrix known from axisymmetric configurations) by a thin layer around the perturbed separatrix. Because of the deformation of this thin layer caused by the perturbed separatrix, the connection length of field lines is slightly increased (light blue in figures 2 (b) and (c)). The relative long toroidal width of $\sim 100 \mathrm{deg}$ is caused by the 
On gas flow effects in $3 D$ edge transport simulations for DIII-D plasmas with resonant magnetic perturbation
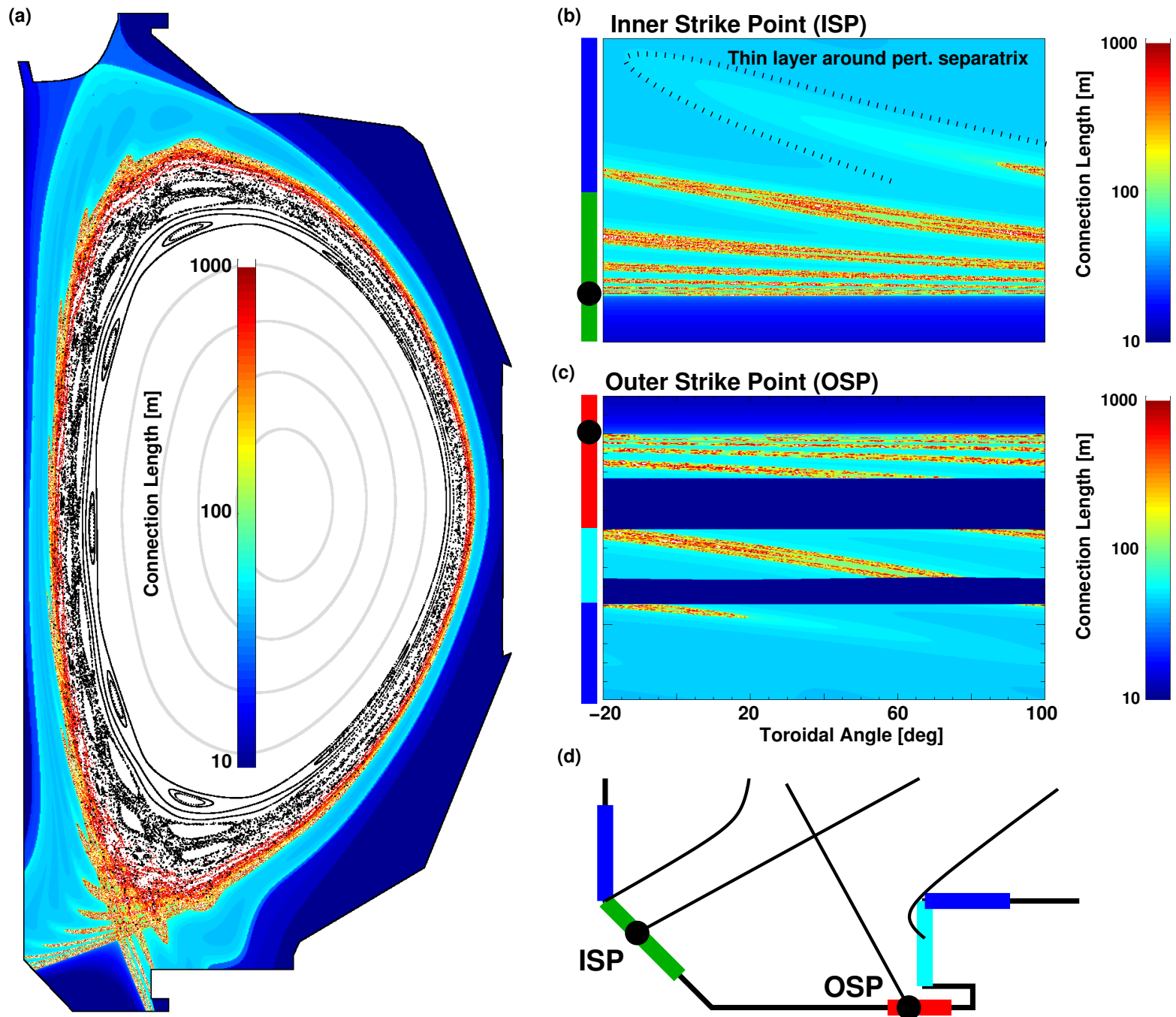

(c)
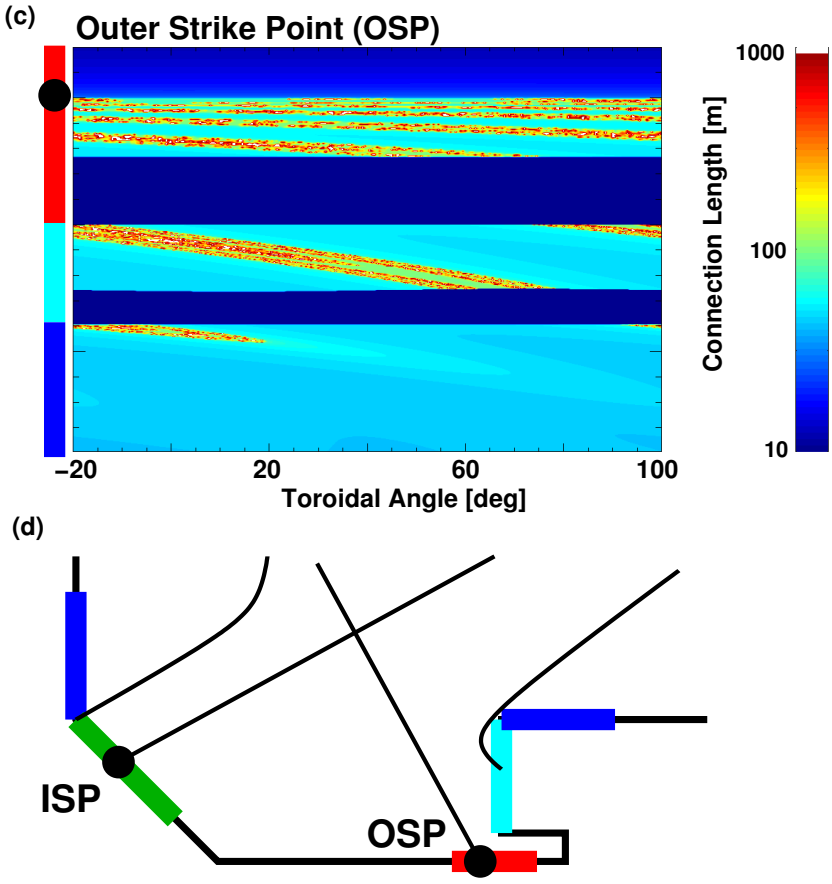

Figure 2. Magnetic field structure of a typical DIII-D plasma with ITER similar shape in the presence of an $n=3 \mathrm{RMP}$ field. The wall-to-wall connection length $L_{c}$ of magnetic field lines is indicated by color. (a) cross-section at the toroidal position $\varphi=0$ deg with Poincaré plot in black, (b) and (c) footprint at the inner and outer strike point, respectively, (d) sketch of the footprint positions on the wall using color bars and a black dot for the position of the unperturbed strike point.

very shallow incident angles of magnetic field lines. The radial width of this thin layer is only a few $\mathrm{mm}$ at the high field side mid-plane.

\subsection{Impact on the plasma density}

We set the re-fuelling rate to $\Gamma_{\text {fuel }}=1.1 \cdot 10^{21} \mathrm{~s}^{-1}$ according to experimental observation. Only NBI is used for re-fueeling during this discharge, which is consistent with the "deep" re-fuelling approximation. Furthermore, we assume $D_{\perp}=0.2 \mathrm{~m}^{2} \mathrm{~s}^{-1}$ throughout the simulation domain and $\chi_{e}=\chi_{i}=3 D_{\perp}$ based on earlier simulations [20]. Simulations have been performed for several pumping efficiencies from $\varepsilon_{\text {pump }}=0.1$ up to $\varepsilon_{\text {pump }}=1.0$ (but the strike points remain at the same positions and the total pumping rate is fixed 


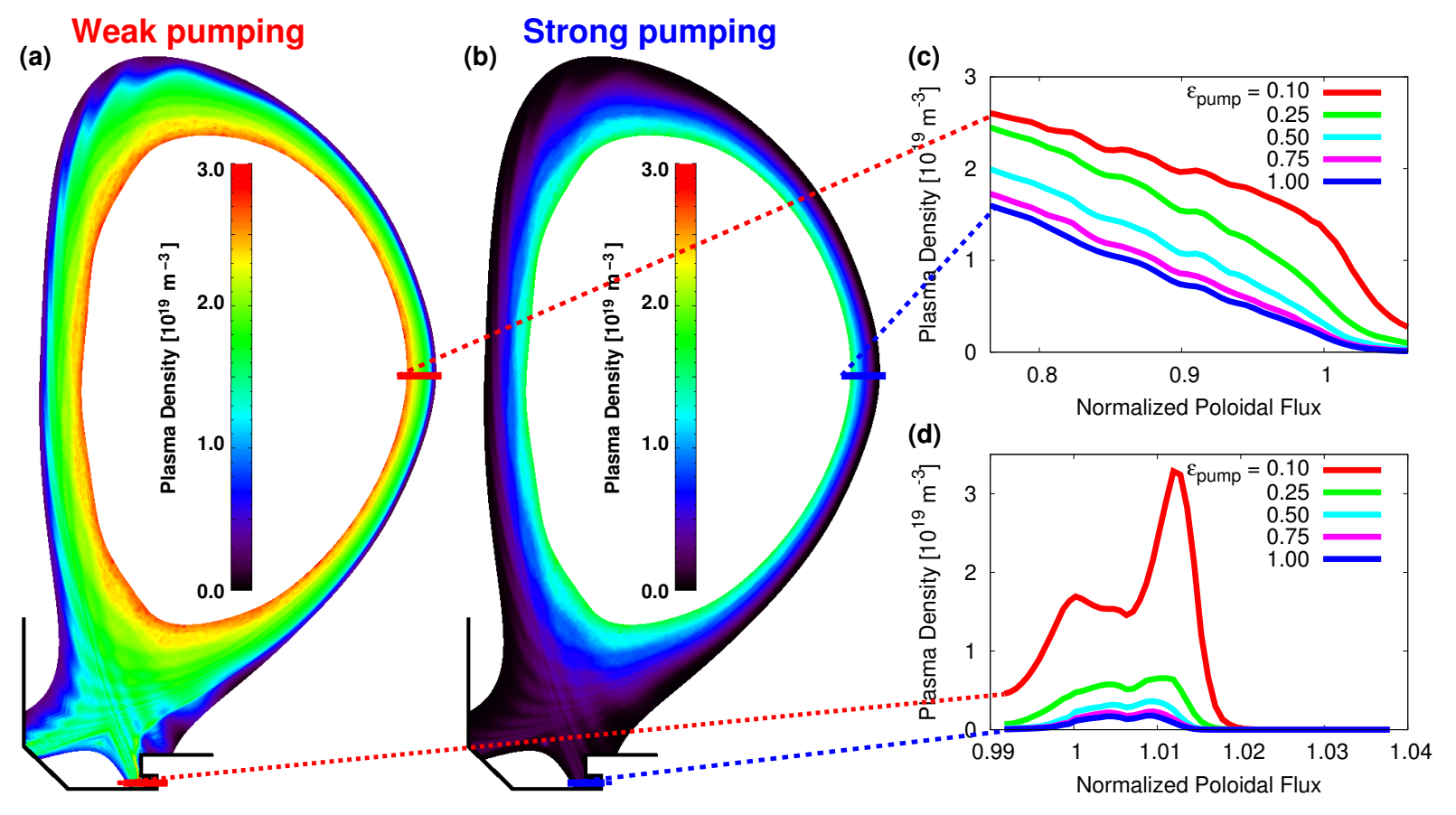

Figure 3. $2 \mathrm{D}$ cuts of the plasma density as a result of weak pumping $\left(\varepsilon_{\text {pump }}=0.1\right)$ (a) and strong pumping $\left(\varepsilon_{\text {pump }}=1.0\right)(b)$. Radial profiles at the low field side (LFS) mid-plane (c) and at the outer strike point (OSP) (d).

$\left.\Gamma_{\text {pump }}=\Gamma_{\text {fuel }}\right)$. An overview of the simulation results for the plasma density is shown in figure 3 (a) and (b). Already a quick comparison demonstrates the significant impact of $\varepsilon_{\text {pump }}$.

Details of the modification of the plasma density due to a variation of $\varepsilon_{\text {pump }}$ is shown in 3 (c) by radial profiles at the low field side (LFS) mid-plane and in (d) by radial profiles at the outer strike point (OSP). A density reduction of $40 \%$ between weak and strong pumping is found at the inner simulation boundary. Note that the present simulations do not account for an edge transport barrier $\left(D_{\perp} \neq\right.$ const is not yet implemented in the code) and hence, that there is no (transport related) pedestal inside the separatrix. Outside the separatrix, the shape of the profiles is determined by recycling sources.

An even larger modification is found in the divertor at the OSP, the plasma density drops by more than one order of magnitude if the pumping efficiency is increased from $\varepsilon_{\text {pump }}=0.1$ to 1.0. The strongest impact is found for low pumping efficiencies, while only a weak modification of the plasma density is found between $\varepsilon_{\text {pump }}=0.75$ and $\varepsilon_{\text {pump }}=1.0$. The pattern of the OSP profiles is related to the striation pattern of the magnetic footprint in figure 2 (c). A noticeable feature is that the density peaks for high pumping efficiencies are almost equal, while the outer peak (just in front of the pump duct) is for low pumping efficiencies almost a factor of 2 larger than the first peak at the strike location of the unperturbed separatrix (i.e. at normalized poloidal flux $\left.\Psi_{N}=1\right)$. The reason is that more neutral particles can accumulate in the pump duct 
On gas flow effects in $3 D$ edge transport simulations for DIII-D plasmas with resonant magnetic perturbation
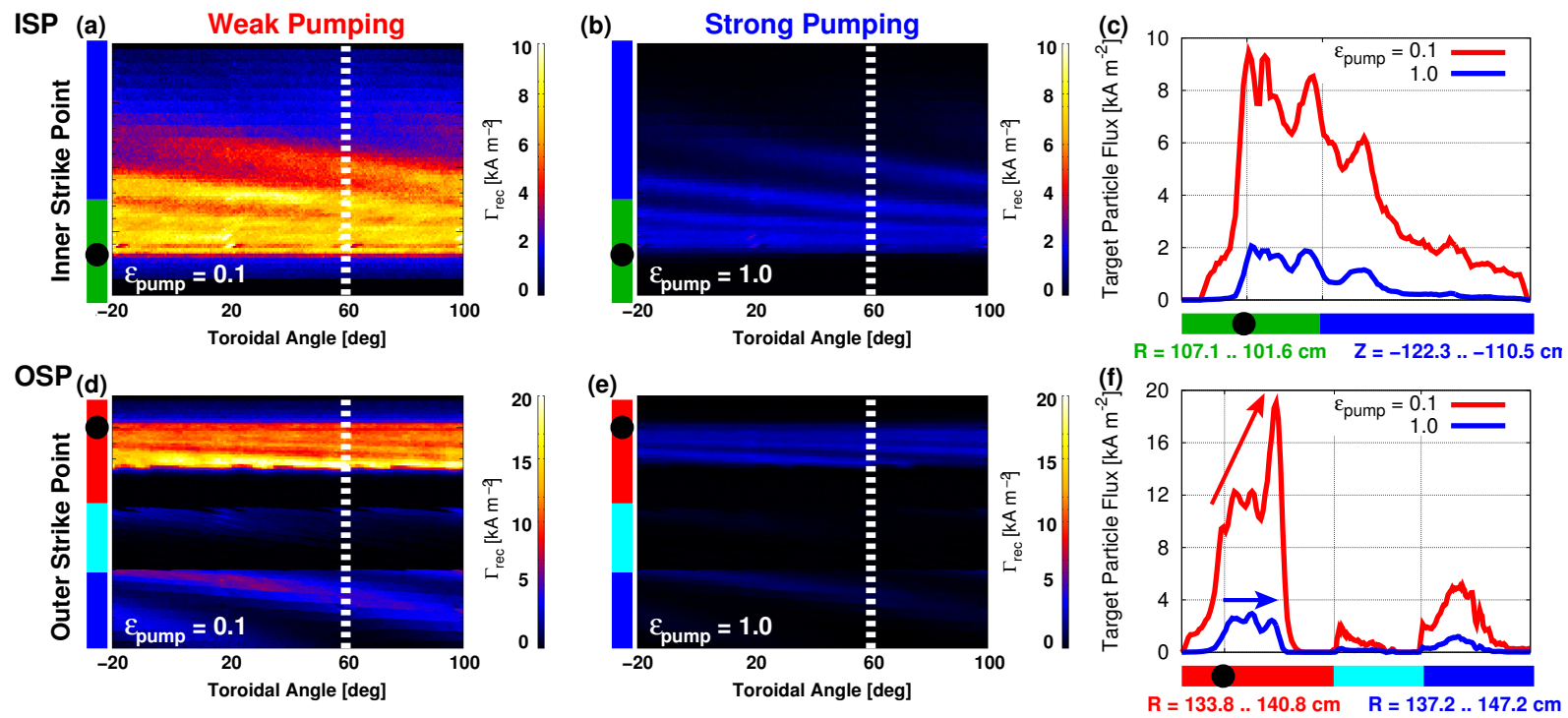

Figure 4. Comparison of the target particle flux distribution for weak (a,d) and strong pumping (b,e) at the inner strike point (upper row) and outer strike point (lower row). Profiles are extracted at $\varphi=60 \mathrm{deg}$ in (c) and (f).

region in the case of low pumping efficiency, and can therefore give a larger contribution to plasma sources just outside the pump duct before they can be pumped.

\subsection{Impact on the target particle flux}

A strong reduction of the target particle flux for high pumping efficiencies is found both at the inner and outer strike points (see figure 4) and is consistent with the density drop. The total target flux is reduced by $85 \%$ from $\varepsilon_{\text {pump }}=0.1$ to $\varepsilon_{\text {pump }}=1.0$. Furthermore, the reduction of the density peak just in front of the pump duct is reflected in the target particle flux (indicated by the arrows in figure 4 (f)). Unfortunately, this outer peak is already out of the range of the Langmuir probes of the experiment and can therefore not be related to experimental observations. This peak asymmetry might affect the carbon sputtering and emission that is observed by a camera during experiments. As plasma impurities have not been included in the present simulations, this comparison is left for future analysis.

The reason for the strong drop of target particle flux is that the prescribed pumping rate $\Gamma_{\text {pump }}$ is connected to the recycling flux $\Gamma_{\text {rec }}$, and that for high pumping efficiencies a lower $\Gamma_{\text {rec }}$ is required to maintain the given $\Gamma_{\text {pump }}$. Although the pumping efficiency $\varepsilon_{\text {pump }}$ is not equal to the ratio $\Gamma_{\text {pump }} / \Gamma_{\text {rec }}\left(\varepsilon_{\text {pump }}\right.$ is the absorption probability for particles at the pump surface, see section 2.1), this ratio is directly correlated to $\varepsilon_{\text {pump }}$ (see figure 5. Unfortunately, the total recycling flux is not measured in the experiment, but e.g. in [28] it is estimated from the core density, the confinement time and some generic "recycling coefficient". The two former quantities are related to the upstream particle flux, while the latter essentially gives an amplification factor for the divertor recycling 
On gas flow effects in $3 D$ edge transport simulations for DIII-D plasmas with resonant magnetic perturbation

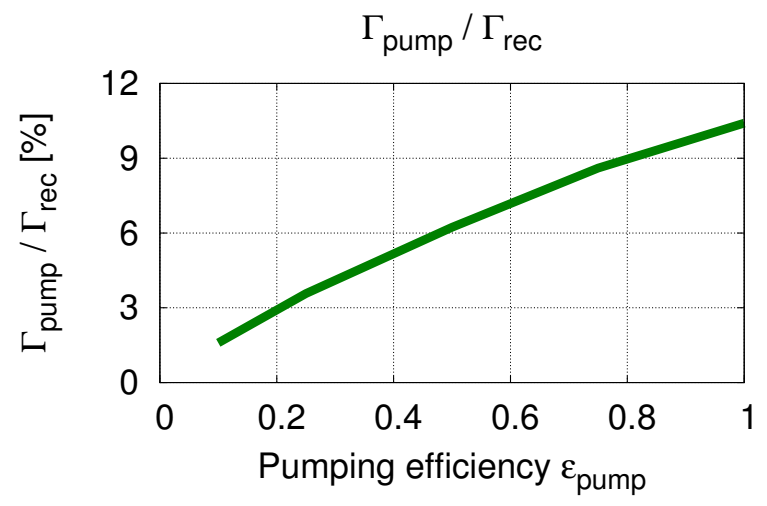

Figure 5. Dependency of the pumping rate to recycling flux ratio $\Gamma_{\text {pump }} / \Gamma_{\text {rec }}$ on the pumping efficiency $\varepsilon_{\text {pump }}$.

flux. Experimental observations are in the range of $\Gamma_{\text {pump }} / \Gamma_{\text {rec }}=2-9 \%$ depending on the plasma shape and edge safety factor [28]. If we include the experimentally observed wall pumping of $2-3 \%$ (no explicitly taken into account in the simulations) in an effective pumping loss fraction of $\Gamma_{\text {pump }} / \Gamma_{\text {rec }} \approx 5-6 \%$ for an ISS plasma at $q_{95}=3.6$, then figure 5 allows to estimate $\varepsilon_{\text {pump }} \approx 0.4-0.5$. Note, however, that a reliable estimation of the numerical parameter $\varepsilon_{\text {pump }}$ can only be given by a more reliable measurement of the total recycling flux $\Gamma_{\text {rec }}$.

Another estimation of $\varepsilon_{\text {pump }}$ can be given from local measurements by Langmuir probes from a strike point sweep in a different phase of the selected discharge. Peak values of the level $50-55 \mathrm{kA} \mathrm{m}^{-2}$ are observed. It can be seen in figure 4 (f) that this is closer to the strong pumping limit, which is consistent with moderately high $\varepsilon_{\text {pump }}$ considering the divertor densities in figure 3 (d). However, one has to keep in mind that, once the strike point is moved away from the pump, the particle flux might increase in order to maintain a prescribed $\Gamma_{\text {pump }}$. Hence, $\varepsilon_{\text {pump }}$ could be even closer to the strong pumping limit for the given magnetic configuration in the simulations. Both estimations suggest that $\varepsilon_{\text {pump }}$ is not too small, and hence, in a range in which its impact on the edge plasma is not too strong.

\subsection{Impact on the target heat flux}

Contrary to the target particle flux, there is no significant impact of the pumping efficiency on the target heat flux (see figure 6). Considering that the distribution of heat flux is primarily given by the magnetic footprint (which does not change), this result is directly linked to total power balance. The total input power into the plasma edge of $P_{\text {heat }}=6.3 \mathrm{MW}$ is taken from the corresponding experimental observations, already accounting for core power losses. In both cases, i.e. weak and strong pumping, edge power losses due to interactions with neutral particles stay at a level of $10-20 \%$ of the input power. As a consequence, also local heat fluxes are similar in both cases. 
On gas flow effects in 3D edge transport simulations for DIII-D plasmas with resonant magnetic perturbation
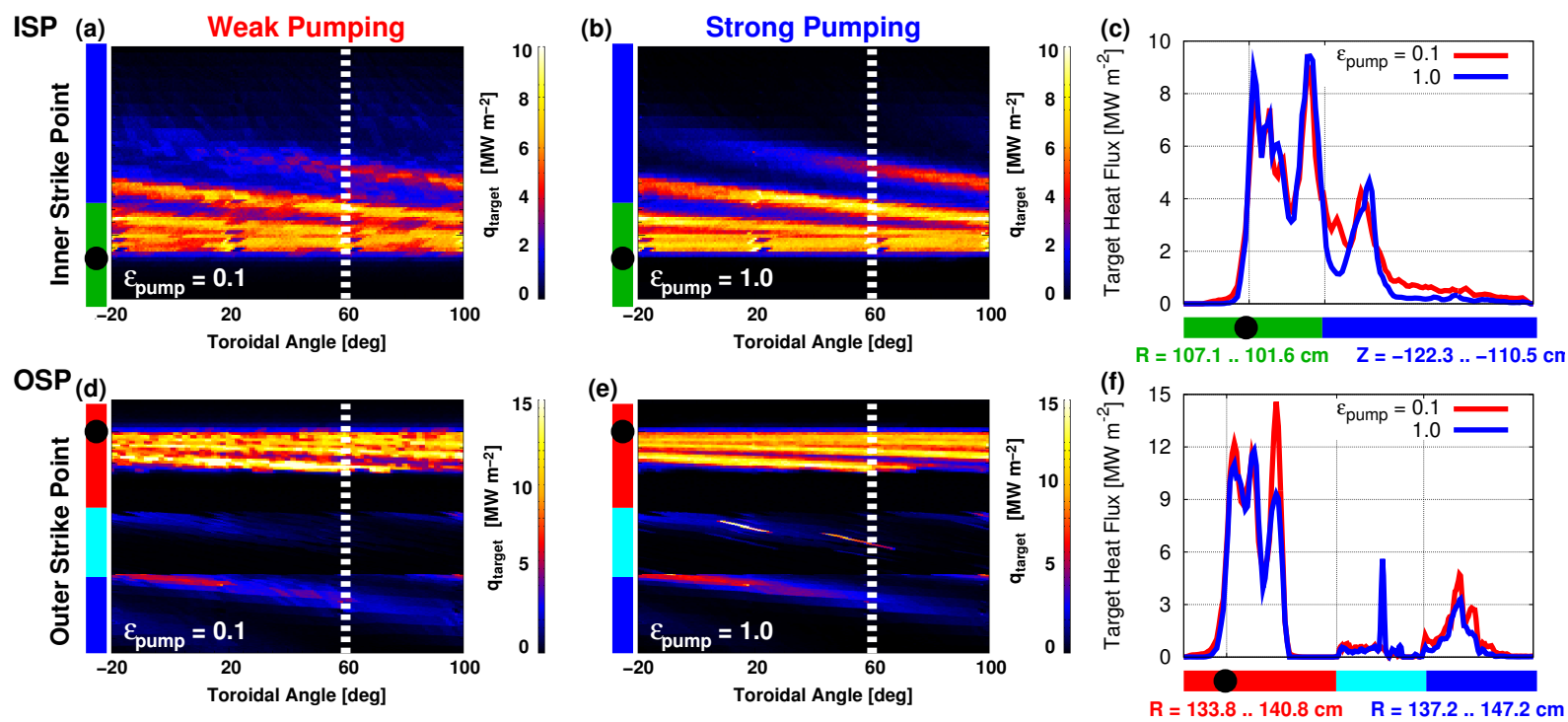

Figure 6. Comparison of the target heat flux distribution for weak (a,d) and strong pumping (b,e) at the inner strike point (upper row) and outer strike point (lower row). Profiles are extracted at $\varphi=60 \mathrm{deg}$ in (c) and (f).

(a)

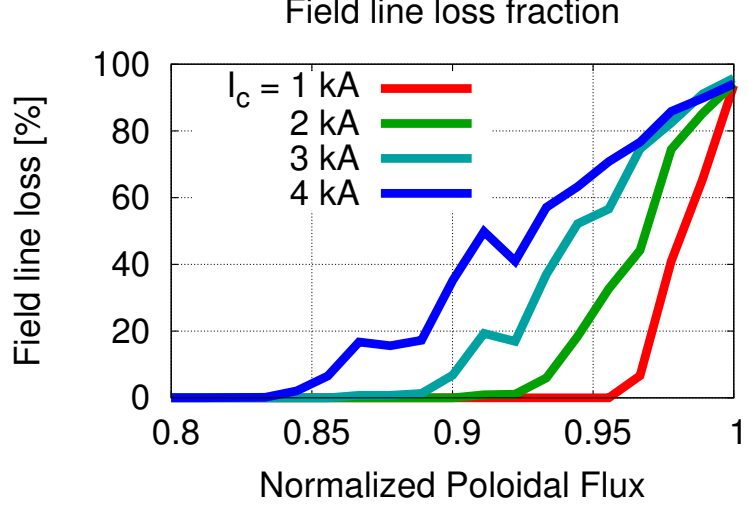

(b)

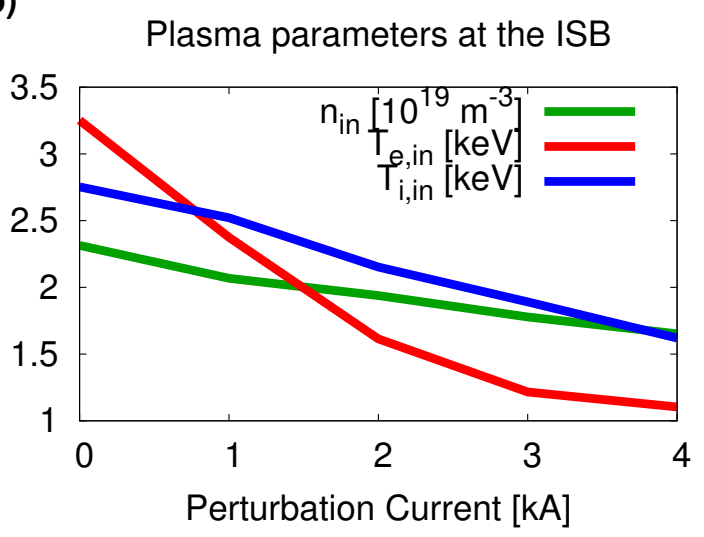

Figure 7. (a) Field line loss fraction for increasing perturbation current $I_{c}$. (b) Plasma parameters at the inner simulation boundary for increasing $I_{c}$.

\section{Modification of edge transport with increasing $I_{c}$}

We keep the fuelling rate fixed at $\Gamma_{\text {fuel }}=1.1 \cdot 10^{21} \mathrm{~s}^{-1}$ (as it has been done in the corresponding experiment) and study the impact of an increasing perturbation current on the plasma edge, in particular at the inner simulation boundary. Furthermore, we assume optimal pumping, i.e. $\varepsilon_{\text {pump }}=1.0$ in the following simulations. The "openness" of the plasma edge can be characterized by the field line loss fraction $\Xi\left(\Psi_{N}\right)$ for a given radial position. $\Xi\left(\Psi_{N}\right)$ is calculated by distributing initial points in toroidal and poloidal direction on an unperturbed flux surface, and by field line tracing from these initial points and counting the amount of field lines lost to the wall. It can be seen in 
figure 7 (a) that at a given radial position $\Psi_{N}$, an increasing amount of field lines is lost to the wall with increasing $I_{c}$. Furthermore, field lines are lost from deeper within the plasma with increasing $I_{c}$. Both results in an increasing outward transport due to plasma transport along open field lines.

As a consequence, edge plasma parameters are reduced with increasing $I_{c}$, e.g. a density drop of $-30 \%$ is found at the inner simulation boundary for the full perturbation current $I_{c}=4 \mathrm{kA}$ compared to the reference case without RMPs (see figure $7(\mathrm{~b})$ ). This density reduction is indeed observed in the corresponding experiment [31] $(-40 \%)$ and is referred to as "particle pump-out". In this particular experiment, a reduction of $-20 \%$ in the electron temperature is observed, which is significantly different from the strong temperature drop of $-65 \%$ in the simulations. One reason for this mismatch could be that the parallel electron heat conduction is overestimated in the fluid ansatz for the edge plasma. In particular, the impact of $I_{c}$ on the ion temperature is much weaker, consistent with much smaller parallel ion heat conductivities. Furthermore, a modification of the magnetic field structure due to a plasma response - in particular a reduction of the field line loss fraction - would probably mitigate the temperature drop, but also the density drop. In addition, the assumption of constant cross-field heat (and particle) transport coefficients in space and during the $I_{c}$ scan might be too simple. That an edge transport barrier which is modified by RMPs is an important ingredient for the explanation of the observed phenomena has been indicated in [29, 30], and therefore suggests that further refinements of our model are necessary.

The increasing field line losses for increasing $I_{c}$ are related to the formation of a striation pattern in the magnetic footprint. The formation of such a striation pattern in the target particle and heat fluxes is demonstrated in figure 8 (d) and (e), respectively, for the inner strike point. As already indicated in the magnetic footprint in figure 2 (b), the striation pattern is extended beyond the boundary given by the perturbed separatrix. While the deformation of a thin layer around the separatrix results only in a weak increase of the connection length (from $\sim 47 \mathrm{~m}$ to $\sim 54 \mathrm{~m}$ ), cross-field transport into this layer leads to a significant level of target particle and heat fluxes. This can be seen by the profiles in figure 8 (a)-(c) for the outer peak at $\Delta l \approx 7 \mathrm{~cm}$ from the unperturbed strike point. The particle flux at this peak is still $\sim 55 \%$ of the main peaks for $I_{c}=4 \mathrm{kA}$. Even for small perturbation currents $\left(I_{c}=1 \mathrm{kA}\right)$, an extended peak of $\sim 60 \%$ of the main peak particle flux is found at $\Delta l=3.5 \mathrm{~cm}$ (i.e. already in the regular SOL).

The level of this extended peak is dependent on the selected level of cross-field transport. We have chosen $D_{\perp}=0.2 \mathrm{~m}^{2} \mathrm{~s}^{-1}$ and $\chi_{e}=\chi_{i}=3 D_{\perp}$, which is low enough to produce a pronounced striation pattern [20] and should give a conservative estimate of the strike point extension. The general formation of a striation pattern of the heat flux is similar to that of the particle flux. However, the extended heat flux peak for $I_{c}=4 \mathrm{kA}$ is only $\sim 30 \%$ of the main peak. The reason for the relative smaller heat flux peak is the fast parallel electron heat transport, resulting in a smaller cross-field to parallel transport ratio for heat than for particles. 
On gas flow effects in 3D edge transport simulations for DIII-D plasmas with resonant magnetic perturbation
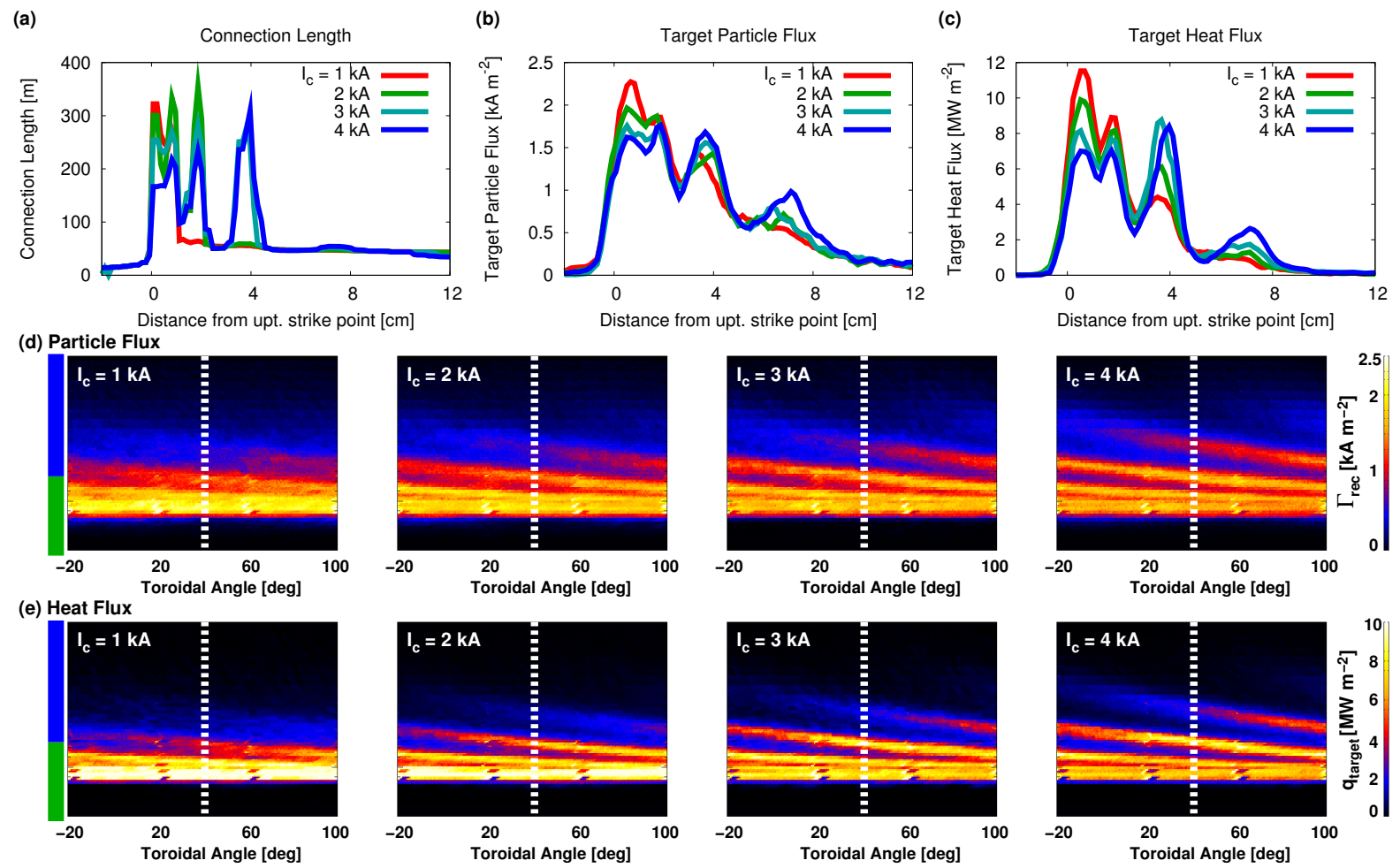

Figure 8. Formation of a striation pattern at the inner strike point. Profiles of the connection length $L_{c}$ at $\varphi=40 \mathrm{deg}$ (a). The corresponding profiles of particle flux $\Gamma_{\text {rec }}(\mathrm{b})$ and heat flux $q_{\text {target }}(\mathrm{c})$ are extracted from (d) and (e).

The simulation results show peak heat fluxes of the order $7-10 \mathrm{MW} \mathrm{m}^{-2}$ which is much larger than experimental observations (which are of the order $3-6 \mathrm{MW} \mathrm{m}^{-2}$ $[31,32])$. These large heat fluxes are related to the low fraction of $10-20 \%$ edge power losses caused by radiation and ionization of neutral hydrogen. Considering an average strike point width of $\Delta r_{\text {ISP }} \approx 6 \mathrm{~cm}$ at $R \approx 102 \mathrm{~cm}$ and $\Delta r_{\mathrm{OSP}} \approx 3 \mathrm{~cm}$ at $R \approx 135 \mathrm{~cm}$ and an input power of $P_{\text {heat }}=6.3 \mathrm{MW}$, heat fluxes of $\bar{q} \approx 10 \mathrm{MW} \mathrm{m}^{-2}$ can indeed be expected. Impurity production and transport (which has been neglected so far) might provide an additional power loss channel and hence, result in lower target heat fluxes closer to experimental observations.

\section{Conclusions}

The EMC-EIRENE code has been advanced to include the effects of neutral gas pumping and core re-fuelling for a closer match to experimental conditions. The implementation of pumping introduces a new model parameter, the pumping efficiency $\varepsilon_{\text {pump. }}$. It has been shown that this parameter has a significant impact on divertor parameters such as density and target particle flux, which is related to the direct link between recycling flux and pumping rate. However, an estimation of $\varepsilon_{\text {pump }}$ guided by experimental observations suggests that a realistic value for $\varepsilon_{\text {pump }}$ is in a range in which its impact on the edge 
On gas flow effects in $3 D$ edge transport simulations for DIII-D plasmas with resonant magnetic perturbation

plasma is not too strong.

The fueling rate has been taken from experimental observations and used as boundary condition for simulations based on different perturbation currents. It has been shown that an increasing perturbation current results in a density drop of $30 \%$ between the full perturbation scenario and the reference case without perturbations, which is in general agreement with experimental observations. On the other hand, a strong drop of electron temperature is found in the simulations, which is probably caused by an overestimation of parallel electron heat conduction in the fluid model. Further model refinements should also include the modification of the magnetic field structure due to a plasma response and a modification of cross-field transport with increasing perturbations.

We have shown that a striation pattern in the target particle and heat fluxes emerges with increasing perturbation current. However, this striation pattern is extended into the regular SOL (i.e. beyond the boundary of the perturbed separatrix), which is caused by cross-field transport into a thin layer around the perturbed separatrix.

\section{Acknowledgments}

This work is supported in part by the U.S. Department of Energy under DE-FC0204ER54698.

\section{References}

[1] Huysmans G, 2005 Plasma Phys. Control. Fusion 47 B165. doi:10.1088/0741-3335/47/12B/S13

[2] Evans T E, et al., 2004 Phys. Rev. Lett. 9223 235003. doi:10.1103/PhysRevLett.92.235003

[3] Evans T E, et al., 2006 Nature Physics 2 419. doi:10.1038/nphys312

[4] Liang Y, et al., 2007 Phys. Rev. Lett. 98265004 1. doi:10.1103/PhysRevLett.98.265004

[5] Loarte A, et al., 2002 Plasma Phys. Control. Fusion 44 1815. doi:10.1088/0741-3335/44/9/303

[6] Feng Y, et al., 1997 Journal of Nuclear Materials 241-243 930. doi:10.1016/S0022-3115(97)801687

[7] Feng Y, et al., 1999 Journal of Nuclear Materials 266-269 812. doi:10.1016/S0022-3115(98)008447

[8] Reiter D, 1992 The EIRENE Code, Version: Jan. 92 Users Manual, Berichte des Forschungszentrum Jülich, 2599, ISSN 0366-0885 edition

[9] Reiter D, et al., 2005 Fusion Science and Technology 472172

[10] Feng Y, et al., 2006 Nuclear Fusion 46 807. doi:10.1088/0029-5515/46/8/006

[11] Kobayashi M, et al., 2007 Journal of Nuclear Materials 363-365 $294 . \quad$ doi: 10.1016/j.jnucmat.2007.01.038

[12] Feng Y, et al., 2008 Nuclear Fusion 48 024012. doi:10.1088/0029-5515/48/2/024012

[13] Feng Y, et al., 2009 Nuclear Fusion 49 095002. doi:10.1088/0029-5515/49/9/095002

[14] Kobayashi M, et al., 2004 Contrib. Plasma Phys. 44 1-3 25. doi:10.1002/ctpp.200410003

[15] Kobayashi M, et al., 2004 Nuclear Fusion 44 S64. doi:10.1088/0029-5515/44/6/S07

[16] Harting D, et al., 2008 Contrib. Plasma Phys. 48 1-3 99. doi:10.1002/ctpp.200810017

[17] Frerichs H, et al., 2008 "3D numerical analysis of magnetic topology and edge transport for RMP limiter scenarios at TEXTOR and DIII-D" 35th EPS Conference on Plasma Phys. Hersonissos, 9-13 June 2008 ECA Vol. 32D, P-2.009 http://epsppd.epfl.ch/Hersonissos/pdf/P2_009.pdf

[18] Kobayashi M, et al., 2007 Nuclear Fusion 47 61. doi:10.1088/0029-5515/47/2/001 
On gas flow effects in $3 D$ edge transport simulations for DIII-D plasmas with resonant magnetic perturbation

[19] Frerichs H, et al., 2010 Comp. Phys. Commun. 181 61. doi:10.1016/j.cpc.2009.08.016

[20] Frerichs H, et al., 2010 Nuclear Fusion 50 034004. doi:10.1088/0029-5515/50/3/034004

[21] Frerichs H, 2010 "3D plasma transport in open chaotic magnetic fields: A computational assessment for tokamak edge layers". Technical Report Jül-4321, Berichte des Forschungszentrum Jülich. ISSN 0944-2952

[22] Harting D, et al., 2011 "Validating the 3D edge code EMC3-EIRENE against 2D simulations with EDGE2D-EIRENE for JET single null configurations" Journal of Nuclear Materials in press doi:10.1016/j.jnucmat.2011.01.030

[23] Feng Y, et al., 2011 Plasma Phys. Control. Fusion 53 024009. doi:10.1088/0741-3335/53/2/024009

[24] Lunt T, 2011 Nuclear Fusion, Proceedings of the 5th SFP Workshop 2011

[25] Braginskii S, 1965 Review of Plasma Physics 1205

[26] Evans T et al., 2005 Journal of Physics: Conference Series 7174 doi:10.1088/1742-6596/7/1/015

[27] Wingen A et al., 2009 Phys. Plasmas 16042504 doi:10.1063/1.3099053

[28] Unterberg E A, et al., 2010 Nuclear Fusion 50 034011. doi:10.1088/0029-5515/50/3/034011

[29] Tokar M, et al., 2008 Phys. Plasmas 15 072515. doi:10.1063/1.2959122

[30] Rozhansky V, et al., 2010 Nuclear Fusion 50 034005. doi:10.1088/0029-5515/50/3/034005

[31] Schmitz O, et al., 2008 Plasma Phys. Control. Fusion 50 124029. doi:10.1088/0741$3335 / 50 / 12 / 124029$

[32] Jakubowski M, et al., 2009 Nuclear Fusion 49 095013. doi:10.1088/0029-5515/49/9/095013 